\title{
Preprocessing MRI Images of Colorectal Cancer
}

\author{
Srivaramangai $\mathbf{R}^{1}$, Prakash Hiremath ${ }^{2}$ and Ajay S Patil ${ }^{3}$ \\ ${ }^{1}$ Department of Information Technology, University of Mumbai \\ Mumbai, Maharashtra 400098, India \\ ${ }^{2}$ Department of Computer Science, KLE Technological University \\ Hubli, Karnataka 580031, India \\ ${ }^{3}$ School of Computer Sciences, North Maharashtra University \\ Jalgaon, Maharashtra 425001, India
}

\begin{abstract}
The precision cancer diagnosis is possible owing to the sophisticated technologies based digital image processing tools. Among the various imaging modalities, Magnetic Resonance Imaging (MRI) is of paramount interest, especially for colorectal cancer imaging. Despite the fact that MRI is a superior technology, an MRI image does contain artifacts and distorted signals. Numerous algorithms and approaches have been studied and implemented for various cancer diagnostics. Yet, more augmented techniques need to be developed, since the study is complex and needs a maximum possible accuracy of detection. In this paper, the research work focuses on the various preprocessing techniques such as noise removal techniques and image enhancement techniques. These methods are analyzed for their performance using statistical parameters and the optimal method is determined for generating a noise-free edgesharp intensity enhanced MRI images of colon and rectum cancer, paving for precision diagnosis. The experimental results are analyzed in terms of various image quality metrics.
\end{abstract}

Keywords: Preprocessing, colorectal cancer, contrast enhancement, sharpening, denoising, filtering, image quality metrics.

\section{Introduction}

The design of computer-assisted medical diagnostics, especially for early detection of various types of cancer in patients, involves extensive application of digital image processing techniques. Though many techniques have been developed for automated cancer diagnosis, still it is niche area due to the higher accuracy level expected of these systems. Colorectal cancer is the third biggest threat worldwide and it requires superior automated tools for its early detection and subsequent treatment plan. The main modalities of medical imaging of colon and rectum region are CT, PET, MRI and X-Ray. The digital images acquired through such modalities are prone to noise and possibilities of artifacts, which may be due to the modality itself or due to the particles present in the environment between the imaging device and the scanned human body. The presence of such artifacts may lead to an improper diagnosis of the diseases. This is because the features extracted from the regions of interest (ROI) do not represent the legion more precisely and thus fail to detect the existence of malignity. Thus it is imperative to have effective preprocessing step for treating such images for noise removal and image enhancement. To have a more justification on the need of preprocessing, one has to look into the details of image acquisition. In any imaging modality, whether CT, PET or MRI, the images normally consist of artifacts which may be due to one of the following reasons: patient-specific artifacts which occur due to metal artifacts, hardening of motion beam; the processing method and equipment based artifacts are usually caused due to partial volume effect, ring and staircase [1]. Hence, it is essential that these images undergo preprocessing operations before being considered for diagnosis.

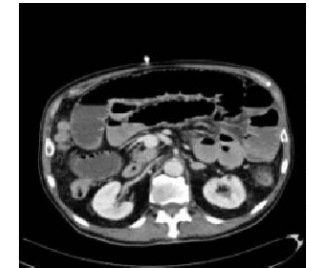

(a)

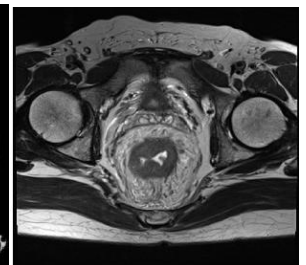

(b)
Fig 1: Blurred and noisy MRI images of (a) colon and (b) rectum cancer (Courtesy : Radiopaedia) 
Figure 1 shows a colon and a rectum MRI image which are noisy and blurred. With this image quality, it will be difficult for an automated system to draw inference. Several research efforts that address this issue have been reported in the literature survey for removing the noises, artifacts and enhancing the brightness and contrast of images of modalities like CT, X-RAY as well as MRI for various types of medical images. In this, the objective is to develop a better method of noise and artifacts removal and thus achieve image enhancement of MRI images of colon and rectum cancer.

\section{Concepts of Preprocessing}

In digital image processing, preprocessing of an image involves low level processes such as noise reduction, image enhancement, image sharpening, etc. [1]. Image enhancement is achieved in terms of improving the sharpness, brightness, contrast (if required) by de blurring the image, removing the noise and transforming the colour, hue and intensity levels and, thereby making the image better suited for the application under consideration[2]. When an image is acquired by an imaging device through some modality, the immediate process required is scaling. In particular, the medical images are acquired through modalities like MRI, CT, Colonoscopy, PET, etc. After scaling, the image preprocessing needs to be performed to improve the clarity of the image that facilitates image analysis.

Contrast enhancement is preferred for MRI images, since it is not a color image. Three contrast enhancement techniques, namely, general contrast enhancement, histogram equalization, adaptive histogram equalization, are in popular use. The general contrast adjustments technique maps the intensity values in the input grayscale image with that of the specified values; if low and high values are not specified, then $1 \%$ of data is saturated at low and high intensities of the image. Thus it increases the contrast of the output image. In histogram equalization, the contrast is enhanced by redistribution of pixel values with uniform probability distribution.

In adaptive histogram equalization, the grayscale image is transformed using contrast-limited adaptive histogram equalization (CLAHE). The difference between histogram equalization and adaptive histogram equalization is that in the second one the CLAHE works on small regions in the image may be in tiles or cells, rather than the entire image. So each cell's contrast is enhanced. In order to avoid induced boundaries, the neighboring tiles are then joined using bilinear interpolation.

The contrast enhanced image is further sharpened using high pass filter, which is a frequency domain filter. It is used for sharpening the edges of the image and, at the same time, preserving the information. Next issue to be addressed is noise removal. The reason for noise in the image is either due to acquisition modalities or later when the image gets transmitted. Thus the model of a noisy image can be given as:

$$
\mathrm{Z}(\mathrm{x}, \mathrm{y})=\mathrm{X}(\mathrm{x}, \mathrm{y})+\mathrm{Y}(\mathrm{x}, \mathrm{y})
$$

In Eq.(1), $X(x, y)$ is the original image, $Y(x, y)$ represents the noise in that image and $\mathrm{Z}(\mathrm{x}, \mathrm{y})$ is the resultant noisy image. Noise removal techniques can be broadly divided into approaches based on filtering, wavelet and multifractal. The different noise models are salt and pepper noise, Gaussian noise, Poisson noise and Speckle noise [3]. Since it is difficult to decide by perception, the type of noise in an image, the selection of a particular noise model and the respective denoising technique is purely based on the type of image and its application. There is no generalized approach that can be followed for all type of images and all types of applications. Hence, these methods are required to be tested in order to find the suitable technique for the type of image modality considered for disease diagnostics under study. The nature of different noise types is given below:

\subsection{Salt and Pepper Noise}

It is seen as white and black pixels sparsely. It appears as spots in and around the image. It is generally used as a model to detect the defects in the images during transmission.

\subsection{Gaussian Noise}

Gaussian noise, or random noise, is based on statistics having a probability density function (PDF) as that of Gaussian distribution.

\subsection{Poisson Noise}

This noise, also known as photon noise, has a form of uncertainty when measuring light with respect to photon detections. In bright light, its expected magnitude is dependent on the signal and becomes 
dominant in the image. This is a form of shot noise which follows Poisson distribution.

\subsection{Speckle Noise}

This noise is granular in nature and inherently exists in all types of images taken through various modalities and sources. It degrades the quality of images very apparently and the information is lost.

The filtering techniques, namely, mean filter, median filter and adaptive median filters, are prominently used for denoising medical images. In the mean filter, also known as average filter, a $3 \times 3$ window is used to replace the central pixel value by the average of the nine pixels of the window. It basically helps in reducing the noise and smoothen the image. In the median filter, a $3 \times 3$ window is used to replace the central pixel value by the median of the nine pixels of the window. It removes noise. Further, it overcomes the reduction of image sharpness of mean filter and, hence, suitable for edge detection or region growing. The adaptive median filter can handle both impulse and non impulse noise. Besides smoothening, it also preserves the details of the image. Instead of replacing the centre pixel with the median value, this filter replaces corrupted (noisy) pixels in the window by the median value of the pixels in the filtering window. Adaptive median filter is best suited for low noise densities because those corrupted pixels which undergo replacement are very few. At higher noise densities, the window size has to be increased so that a better noise removal can be achieved.

In this paper, the objective is to investigate preprocessing methods for image enhancement of MRI images of colon and rectum that improve image quality and thus assist digital image analysis based cancer diagnostics. The contrast adjustment, sharpening and noise removal are considered. Different noise models are explored and the statistical parameters of image quality are evaluated to find the optimal method of denoising such images.

\section{Related Work}

There are numerous preprocessing techniques for digital image processing. The most important among those techniques are image enhancement and denoising. In medical imaging applications, image denoising plays a vital role. Despite high technology medical imaging modalities, the need for preprocessing these images persists before any image analysis is undertaken.

\subsection{Noise Removal and Filters}

An efficient noise reduction algorithm proposed for CT images in [4] uses window-based multi-wavelet transformation and thresholding for Gaussian noise removal and image enhancement. Neha et al.[5] bring out the comparative results of various filtering techniques applied on MRI images of brain cancer, which show that max filter performs better than mean, arithmetic mean and min filters. Valarmathy et al. [6] have examined mean, median and adaptive median filters for removal of noise. Geetha and Selvi[7] have done comparative analysis of various denoising methods in terms of peak signal to noise ratio (PSNR), signal to noise ratio (SNR) and validated by visual inspection of the images. In the absence of prior knowledge of the type of noise existing in an image, most research efforts aim for removal of Gaussian noise and often do not give required results. Discrete wavelet transformation algorithms are also developed in the literature for image denoising.

Priyadarshini[8] has proposed a new denoising technique for MRI images of brain which is based on spectral subtraction method. Signal to noise ratio (SNR) has been improved significantly by the proposed technique. The filter referred as $\operatorname{SSD}$ (Spectral Subtraction Denoising) is a filter mainly dependent on the SNR value. Here, the lower SNR components have reduced the virulence than higher SNR components. Wavelet denoising method is used by JecilyJemila and Jayshankar [9], which has enhanced the visual quality of images. This improvement leads to the accuracy of image segmentation. In case of functional MRI, wavelet based denoising methods help in increasing the SNR level, which retains the shape of the region of interest. Rajesh Patil and Bhalachandra [10], have used high pass filter and median filter for noise removal and enhancement, which led to effective brain tumor extraction. It has also used water shed segmentation and threshold segmentation for extracting the brain tumour region. Usage of filters have resulted in good quality, enhanced image of the brain. Siti et. al [11], have used an algorithm for denoising the noisy MRI images, termed as switching AFKM, is proposed for segmentation process and then a conventional clustering algorithm is used for clustering the objects. The 
proposed method minimizes the salt and pepper noise and sharpens the image, thus giving a good quality image output.

Gopinath[12] presented the high pass filter for enhancement of the MRI image of prostate cancer and has used median filter for removing noise. Anuradha et al. [13] have used median filter for removing the salt and pepper noise. Ben George and Karnan[14] proposed a method for preprocessing of MRI image of brain. After comparing median filter, weighted median filter and center weighted median filter, it was found that center weighted median filter yields optimal results for noise removal and image enhancement. Deepa and Sumithra[15] have proposed a different technique which combines the spectral subtraction and wavelet packet based methods. This method has been used to test for the common four types of noises at various decibel levels. The result has shown that it has not only removed the noise but also has maintained the image quality. Deepa and Suganthi [16], have done a performance evaluation of various filters for denoising medical images. A study of noise detection and noise removal techniques for medical images has been done by Bhausaheb et. al. [17], which reveals that the choice of noise inputs and the relevant noise removal filters in medical images, such as MRI, CT Scan, X-ray, depends on the type of noise existing in these images on acquisition and so a general technique cannot be used for noise input and removal. Amutha et al. [18] have presented a spatially adaptive method, namely, optimized single-stage principal component analysis (OSPCA) for improving the signal to noise ratio in MRI images of breast. The information and the clarity of image can't be distorted while using denoising filters. So to protect the data, blocks of similar pixels are grouped together using local pixel grouping (LPG) and the image is transformed in PCA domain. When smoothing is done, shrinking techniques is used so that the information is preserved.

\subsection{Contrast enhancement}

Oak and Kamathe [19] have presented a histogram based approach for contrast enhancement and observed that, for $\mathrm{T} 1$ weighted axial images of brain MRI, modified bi-histogram equalization(BHE) and contrast limited adaptive histogram equalization (CLAHE) give good contrast but still has some disturbed background. Brightness preserving bi-histogram equalization (BBHE) gives good contrast for the same. Similarly, for T2 weighted axial MRI images, CLAHE and BBHE are much more suitable. However, BBHE gives uniform histogram and CLAHE gives clipped histogram. For proton density (PD) weighted images, BBHE or modified $\mathrm{BHE}$ is seen to be the optimal option. Reddy et al. [20] proposed a methodology for detecting the bone cancer by identifying the mean intensity of MRI image. Once malignity is detected based on mean intensity threshold value, the stage of the cancer is also identified. Further, it focuses on detecting the cancer based on tumor size in an MRI image and cancer stage based on mean intensity threshold value.

Nelinkanti et al. [21] proposed a framework to enhance the input MRI image with various image processing techniques and used adaptive k-means clustering technique for segmentation. This segmented image is further analyzed for detecting the presence of colorectal cancer. The calculation of area, mean of the tumor and the minimum distance from tumor to other parts will assist radiologists in finding the stage further. Some researchers have focused on operations other than preprocessing. A new method has been introduced by Harish Kumar et al. [22] for enhancement of mammographic images, which is based on modified mathematical morphology and biorthogonal wavelet transform. The performance of this method is evaluated using contrast improvement index (CII) and edge preservation index (EPI). The results show that the proposed method is better in giving good quality image. Modified tracking algorithm is used by Velusamy et al. [23] for removing the artifacts present in the acquired brain MRI image. This algorithm uses the threshold value of the artifact to remove the disturbances. The filtering technique used here is the centre weighted median filter. Niu et al. [24] have used 2D wavelet packet transform, as a preprocessing method and found it to be more effective in spectral data compression and feature extraction. Histopathological image analysis using image processing techniques has been investigated by Belsare et al.[25], and no specific method is proposed for preprocessing.

KimmiVerma et al. [27] have compared various image preprocessing and segmentation algorithms 
and the choice of the techniques varies according to the type of image acquired. Apart from noise removal, Deshpande et al. [13] have done contrast enhancement of the image. In [27], linear-filtering technique is used by Atiyeh Hashemi and et.al., for enhancing the CT mages of lung cancer. Sheela and Suresh Babu [28] have used skull stripping and histogram equalization for preprocessing the brain MRI images and obtained satisfactory results.

\section{Methodology}

The proposed methodology for preprocessing MRI images of colon and rectum cancer is depicted in the Figure 2 and comprises the following steps:

i. The input MRI images of colon and rectum cancer are converted into gray scale images, which are subjected to contrast enhancement. The image enhancement is measured in terms of statistical parameters of image quality, namely, AMBE, Structural Similarity Index (SSIM), FSIM, Peak to Signal Noise Ratio (PSNR), Mean Square Error (MSE), Signal to Noise Ratio(SNR), CNR.

ii. Image sharpening is performed on the contrast enhanced images using high pass filter.

iii. Noise content is added to the input MRI image and then removed with various filtering techniques. Different proportions of noise, which are modeled as salt-and-pepper, Gaussian, Poisson and speckle noise, are considered.

iv. The noise removal is done using the mean, median and adaptive median filters. The optimal filter is determined based on Mean Square Error (MSE) and Peak to Signal Noise Ratio (PSNR).

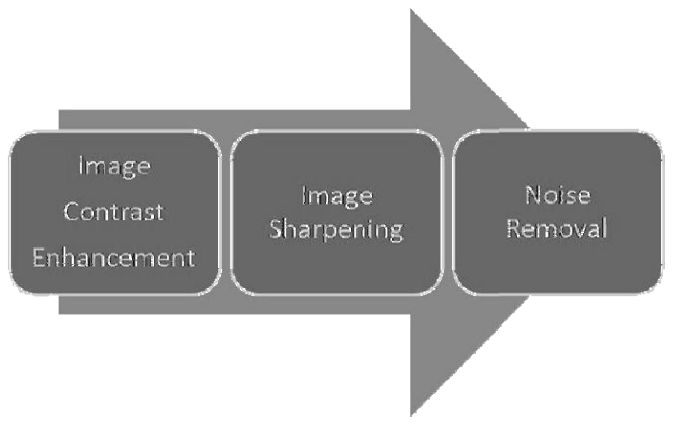

Fig. 2 Block Diagram of the Preprocessing Methodology

The Table 1 describes the various performance metrics along with the degree, wherein $\mathrm{L}$ indicates that the value should be low and $\mathrm{H}$ indicates the value should be high. The optimal $t$ echnique at each step is chosen based on these metrics levels.

Table 1: Image Quality Metrics

\begin{tabular}{|c|c|c|c|}
\hline Metrics & Description & Formula & Degree \\
\hline MSE & $\begin{array}{l}\text { MSE is the square } \\
\text { of difference } \\
\text { between original } \\
\text { and reconstructed } \\
\text { image. }\end{array}$ & $\begin{array}{c}\mathrm{MSE}= \\
\frac{1}{m n} \sum_{i=0}^{n} \sum_{i=0}^{n-1} \| \\
\mathrm{f}(\mathrm{i}, \mathrm{j})-\mathrm{g}(\mathrm{i}, \mathrm{j}) \|^{2}\end{array}$ & L \\
\hline SNR & $\begin{array}{l}\text { Signal to Noise } \\
\text { Ratio is the ratio } \\
\text { of the power of } \\
\text { signal of the } \\
\text { original image to } \\
\text { that of the power } \\
\text { of signal of noisy } \\
\text { image or } \\
\text { reconstructed } \\
\text { image. Some } \\
\text { times it is } \\
\text { represented as the } \\
\text { mean of both } \\
\text { signals. While } \\
\text { calculating power, } \\
\text { SNR is } \\
\text { represented in } \\
\text { decibels, where as } \\
\text { in mean ratio it is } \\
\text { represented as a } \\
\text { number. }\end{array}$ & $\begin{array}{c}\mathrm{SNR}= \\
\text { Psignal } / \text { Pnoise }= \\
\mu / \sigma\end{array}$ & $\mathrm{H}$ \\
\hline CNR & $\begin{array}{l}\text { Carrier-to-Noise } \\
\text { Ratio is the ratio } \\
\text { of the power of } \\
\text { the modulated } \\
\text { signal to the } \\
\text { power of the noise } \\
\text { found in the } \\
\text { information signal } \\
\text { after it has been } \\
\text { demodulated. } \\
\text { Here it is power of } \\
\text { the original image } \\
\text { signal and power } \\
\text { of } \\
\text { reconstructed the } \\
\text { image signal. }\end{array}$ & $\begin{array}{c}\mathrm{CNR}= \\
\operatorname{ABS}\left(\mathrm{S}_{\mathrm{f}-} \mathrm{S}_{\mathrm{g}}\right) / \sigma\end{array}$ & $\mathrm{H}$ \\
\hline PSNR & $\begin{array}{l}\text { Peak Signal to } \\
\text { Noise Ratio. This } \\
\text { metric is used to } \\
\text { measure the } \\
\text { quality with that } \\
\text { of the original or } \\
\text { corrupted image. } \\
\text { It is a logarithmic } \\
(\mathrm{dB}) \text { scale which } \\
\text { is the maximum } \\
\text { value of } \\
\text { maximum signal } \\
\text { power. }\end{array}$ & $\begin{array}{l}\text { PSNR }= \\
20 \log _{10}\left(\frac{M A X_{f}}{\sqrt{M S E}}\right) \\
\mathrm{f}=\text { original image } \\
\text { MSE is as } \\
\text { calculated above in } \\
\text { point } 1 \\
\text { MAX }_{\mathrm{f}} \text { is the } \\
\text { maximum signal } \\
\text { value of the original } \\
\text { image }\end{array}$ & $\mathrm{H}$ \\
\hline SSIM & $\begin{array}{l}\text { Structural } \\
\text { Similarity Index } \\
\text { Measure is a } \\
\text { method for } \\
\text { measuring the } \\
\text { similarity between } \\
\text { the original image } \\
\text { and reconstructed }\end{array}$ & $\begin{array}{c}\operatorname{SSIM}(X, Y)= \\
\frac{\left(2 \mu_{\mathrm{x}} \mu_{\mathrm{y}}+\mathrm{C}_{1}\right)\left(2 \sigma_{\mathrm{xy}}+\mathrm{C}_{2}\right)}{\left(\mu_{\mathrm{x}}^{2}+\mu_{\mathrm{y}}^{2}+\mathrm{C}_{1}\right)\left(\sigma_{\mathrm{x}}^{2}+\sigma_{\mathrm{y}}^{2}+\mathrm{C}_{2}\right)}\end{array}$ & $\mathrm{H}$ \\
\hline
\end{tabular}




\begin{tabular}{|c|c|c|c|}
\hline & $\begin{array}{l}\text { image. Here the } \\
\text { original image is } \\
\text { considered as } \\
\text { perfect and the } \\
\text { reconstructed } \\
\text { image is } \\
\text { compared with it }\end{array}$ & & \\
\hline FSIM & $\begin{array}{l}\text { Feature Similarity } \\
\text { Index Measure is } \\
\text { a measure which } \\
\text { looks at the } \\
\text { feature parameters } \\
\text { involved in the } \\
\text { images and } \\
\text { compares for a } \\
\text { match and high } \\
\text { quality of } \\
\text { intensity values. }\end{array}$ & $\begin{array}{c}\text { FSIM }= \\
\frac{\sum_{x \varepsilon \Omega} S_{L}(x) \cdot P C_{m}(x)}{\sum_{x \varepsilon \Omega} P C_{m}(x)}\end{array}$ & $\mathrm{H}$ \\
\hline RFSIM & $\begin{array}{l}\text { Riesz-transform } \\
\text { based Feature } \\
\text { SIMilarity index. } \\
\text { It is computed by } \\
\text { comparing the } \\
\text { feature maps at } \\
\text { key locations } \\
\text { marked by a } \\
\text { feature mask } \\
\text { between two } \\
\text { images. }\end{array}$ & $\begin{array}{c}\text { RFSIM }= \\
\prod_{i=1}^{5} D_{i} \\
\text { where } \\
\mathrm{D}_{\mathrm{i}}= \\
\frac{\sum \sum d_{i}(x, y) \cdot M(x, y)}{\sum \sum M(x, y)}\end{array}$ & $\mathrm{H}$ \\
\hline UQM & $\begin{array}{l}\text { Universal Quality } \\
\text { Measurement }\end{array}$ & $\mathrm{Q}=\frac{1}{M} \sum_{j=1}^{M} Q_{j}$ & $\mathrm{H}$ \\
\hline IFC & $\begin{array}{l}\text { Information } \\
\text { Fidelity Criterion } \\
\text { is used to evaluate } \\
\text { how much } \\
\text { information are } \\
\text { same in original } \\
\text { image and } \\
\text { reconstructed } \\
\text { image }\end{array}$ & $\begin{array}{c}\text { IFC }= \\
\sum_{k \text { sesublowks }} I\left(C^{N_{k} k} ; D^{V_{k} k} \mid S^{N_{k} k}\right)\end{array}$ & $\mathrm{H}$ \\
\hline MAE & $\begin{array}{l}\text { Mean Absolute } \\
\text { Error is the mean } \\
\text { or average of } \\
\text { absolute } \\
\text { difference } \\
\text { between the } \\
\text { original image and } \\
\text { image after } \\
\text { reconstruction }\end{array}$ & $\operatorname{AVG}(\operatorname{abs}(\mathrm{f}-\mathrm{g}))$ & $\mathrm{L}$ \\
\hline AMBE & $\begin{array}{l}\text { Absolute Mean } \\
\text { Brightness Error } \\
\text { is used to } \\
\text { calculate the } \\
\text { absolute value of } \\
\text { the difference } \\
\text { between the mean } \\
\text { error original } \\
\text { image and mean } \\
\text { error of } \\
\text { reconstructed } \\
\text { image }\end{array}$ & $\begin{array}{l}\text { ABS(AVG(MAE1- } \\
\text { AVG(MAE2)) }\end{array}$ & $\mathrm{L}$ \\
\hline
\end{tabular}

\section{Experimental Results}

The experimentation of the proposed methodology is carried out on the image data set consisting of MRI images of colon and rectum of 15 patients, both being the axial T2 weighted images obtained from MRI scan, using MATLAB software. Thus, the dataset contains 30 MRI images (15 colon and 15 rectum images), which have a combination of normal and abnormal tumours varying from the stage $\mathrm{T} 1$ to $\mathrm{T} 4$.

Since the original MRI image is already in black and white format, not much of variation is seen on grayscale conversion. But the images are converted to 8 bit format so that it is easy to process at the low level. The Figures 3(a)-(d) show a sample colon image and its corresponding resultant images obtained by contrast enhancement methods, namely, general contrast, histogram equalization and adaptive histogram equalization. The Figures 4(a)-(d) show a sample rectum image and its corresponding resultant images. The results are compared in terms of the image quality metrics. PSNR value is very high compared to other parameters.

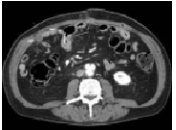

(a)

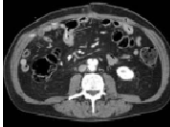

(b)

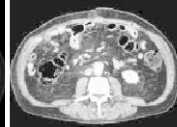

(c)

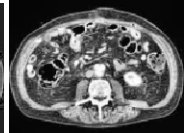

(d)
Fig 3 Colon image: (a) Original image (b) Contrast general (c) Histogram equalization (d) Adaptive histogram equalization.

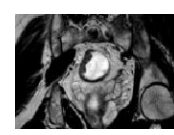

(a)

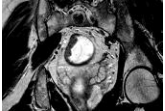

(b)

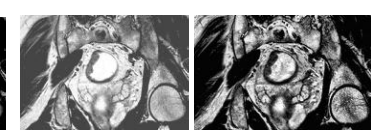

(c) (d)
Fig 4 Rectum image: (a) Original image (b) Contrast general (c) Histogram equalization (d) Adaptive histogram equalization.

By visual inspection, the adaptive histogram equalization yields better image enhancement results. However, image quality needs to be analyzed in terms of some statistical parameters. There are various methods available to evaluate the image quality attained by an image processing technique, which are classified as full reference methods (FR) and no reference methods (NR). The FR methods use a reference image for comparison with the newer image achieved through some process. The NR methods on the contrary do not have any reference image to compare. Usually in image enhancement, the FR methods are used so that the resultant image is evaluated to be better or not. The factors used for such reference are 
sharpness, brightness, contrast, colour, noise, artifacts and distortion. In this paper, FR methods are used for performance evaluation [3] and most of the evaluation parameters mentioned in the Table 1 are computed for images obtained by the three image enhancement methods, namely, contrast adjustment, histogram equalization and adaptive histogram equalization.

The experimental results are presented in the Table 2 for colon and rectum images. It is observed that contrast adjustment method yields higher values for PSNR, SSIM, FSIM, RFSIM, UQM and lower value for $\mathrm{AMBE}$, as compared to the other two methods. Since the contrast adjustment performs better in terms of majority of the metrics, it is a suitable method for image enhancement of MRI images of colon as well as rectum.

Table 2 Comparison of image quality metrics of colon and rectum MRI images obtained by different contrast enhancement techniques-IQM(Image Quality Metrics), CA(Contrast Adjustment), H(Histogram), AH(Adaptive Histogram)

\begin{tabular}{|c|r|r|r|r|r|r|}
\hline \multirow{2}{*}{ IQM } & \multicolumn{3}{|c|}{ Colon Images } & \multicolumn{3}{c|}{ Rectal Images } \\
\cline { 2 - 7 } & \multicolumn{1}{c|}{ CA } & \multicolumn{1}{c|}{ H } & \multicolumn{1}{c|}{ AH } & \multicolumn{1}{c|}{ CA } & \multicolumn{1}{c|}{ H } & \multicolumn{1}{c|}{ AH } \\
\hline SNR & -0.003 & -2.37 & -0.549 & -0.067 & -1.083 & -0.475 \\
\hline CNR & 37.228 & 49.58 & 55.936 & 46.252 & 54.476 & 60.575 \\
\hline AMBE & 1.0443 & 67.85 & 15.329 & 15.234 & 69.729 & 34.476 \\
\hline SSIM & 0.997 & 0.388 & 0.523 & 0.929 & 0.498 & 0.537 \\
\hline \multirow{2}{*}{ PSNR } & 30609. & 11.32 & 19.660 & 26225. & 10.552 & 15.122 \\
& 32 & 5 & & 7 & & \\
\hline RFSIM & 0.993 & 0.250 & 0.268 & 0.849 & 0.297 & 0.120 \\
\hline MAE & 0 & 0.161 & 1.857 & 0.006 & 0.005 & 0.718 \\
\hline UQM & 0.999 & 0.433 & 0.656 & 0.928 & 0.483 & 0.616 \\
\hline \multirow{2}{*}{ IFC } & $3.57 \mathrm{E}-$ & 3.57 & $3.57 \mathrm{E}-$ & $1.87 \mathrm{E}-$ & $1.87 \mathrm{E}-$ & $1.87 \mathrm{E}-$ \\
& 06 & $\mathrm{E}-06$ & 06 & 06 & 06 & 06 \\
\hline FSIM & 0.999 & 0.841 & 0.889 & 0.972 & 0.863 & 0.827 \\
\hline
\end{tabular}

The Figures $5 \mathrm{a}$ and $5 \mathrm{~b}$ shows the comparison of the image quality metrics computed for colon and rectum MRI images using the three types of contrast enhancement techniques and the metric values are the mean of the values obtained for all the colon/rectum images.

\section{Mean values of colon images for contrast Enhancement}

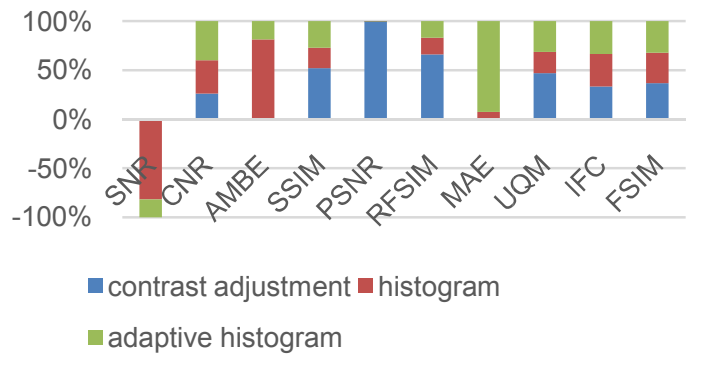

Fig 5a : Comparison of image quality metrics of colon MRI images using the three types of contrast enhancement techniques.
Except CNR, all high degree metrics have higher values yielded by contrast adjustment.

From the Figure $5 \mathrm{a}$ and $5 \mathrm{~b}$, it is clear that contrast adjustment performs better than the other two methods for most of the parameters.

\section{Mean values of Rectum images for Contrast Enhancement}

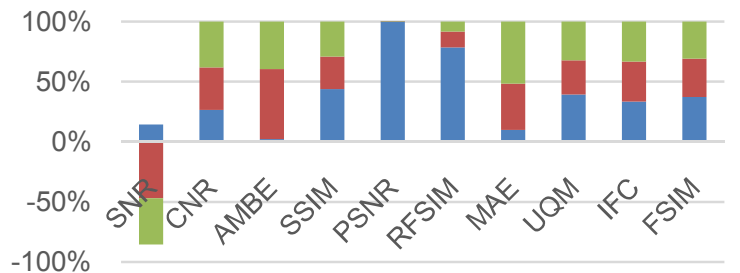

m contrast adjustment $\mathbf{m i s t o g r a m}$ adaptive histogram

Fig 5b : Comparison of image quality metrics of rectum MRI images using the three types of contrast enhancement techniques.

Next, the contrast adjusted images are subjected to image sharpening using high pass filter. The Figure 6 shows samples of one colon image and one rectum image with resultant images obtained by the contrast adjustment followed by sharpening.

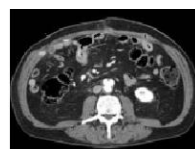

(a)

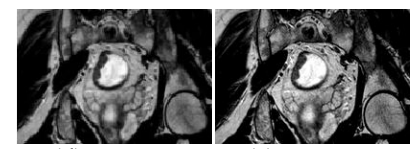

(d)

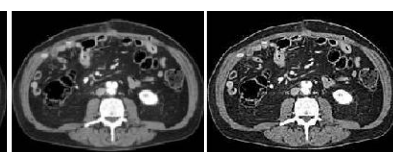

(c)

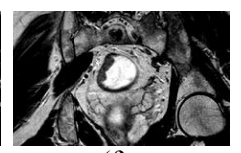

(f)
Fig 6 : Image sharpening: (a) and (d) Original MRI images of colon and rectum; (b) and (e) Contrast enhanced images of colon and rectum in (a) and (d); (c) and (f) Sharpened images of colon and rectum in (b) and (e)

Finally, the sharpened images are subjected to image denoising process. The four noise models, which are in popular use [3][6][9][12], namely, salt-and-pepper(SP), Gaussian (GN), Poisson (PN) and speckle (SK) noise, are considered. The three noise removal filters, namely, mean(MF), median(MDF) and adaptive median(AMDF) filters, are employed and their performance is studied in terms of MSE and PSNR metrics, which are the 
most preferred measures of image quality after noise removal. For salt and pepper, Gaussian and speckle noise, the noise proportions are varied as $5 \%, 10 \%$ and $15 \%$ and then tested with the three filters to remove the added noise along with the inherent noise of that type in the image. In case of Poisson noise, the tool takes the proportion itself within the intensity of the image, and hence specific proportion is not mentioned. The Figure 7 shows the resultant images of denoising by the three filters applied on a sample colon MRI image, while the Figure 8 shows that on a sample rectum MRI image. The experiment is repeated for all the 15 images of colon and 15 images of rectum. While analyzing the metrics, the mean values of MSE and PSNR taken over all the 15 images are considered for each of the noise model, proportion and filter applied.

In order to find the optimal filtering, the peak signal to noise ratio (PSNR) and mean square error (MSE) values of the denoised images are calculated. The PSNR value should be high and MSE value should be low for optimal filtering.
The Table 3 gives the PSNR and MSE values obtained for colon and rectum MRI images using the different noise types with various proportions and noise filters. It is observed that, for colon images with salt and pepper noise, the adaptive median filter yields the highest PSNR and lowest MSE values.

For rectum images with Poisson noise, the mean filter yields the lowest MSE, whereas for that with salt and pepper noise, the adaptive median filter yields highest PSNR value. However, the difference of MSE values corresponding to salt and pepper noise and Poisson noise for rectum image is 0.00173 , which is marginal. Thus, the adaptive median filter can be considered as optimal for rectum images also. Similarly, the optimal filters suitable for removing other noise types are determined and presented in the Table 4.

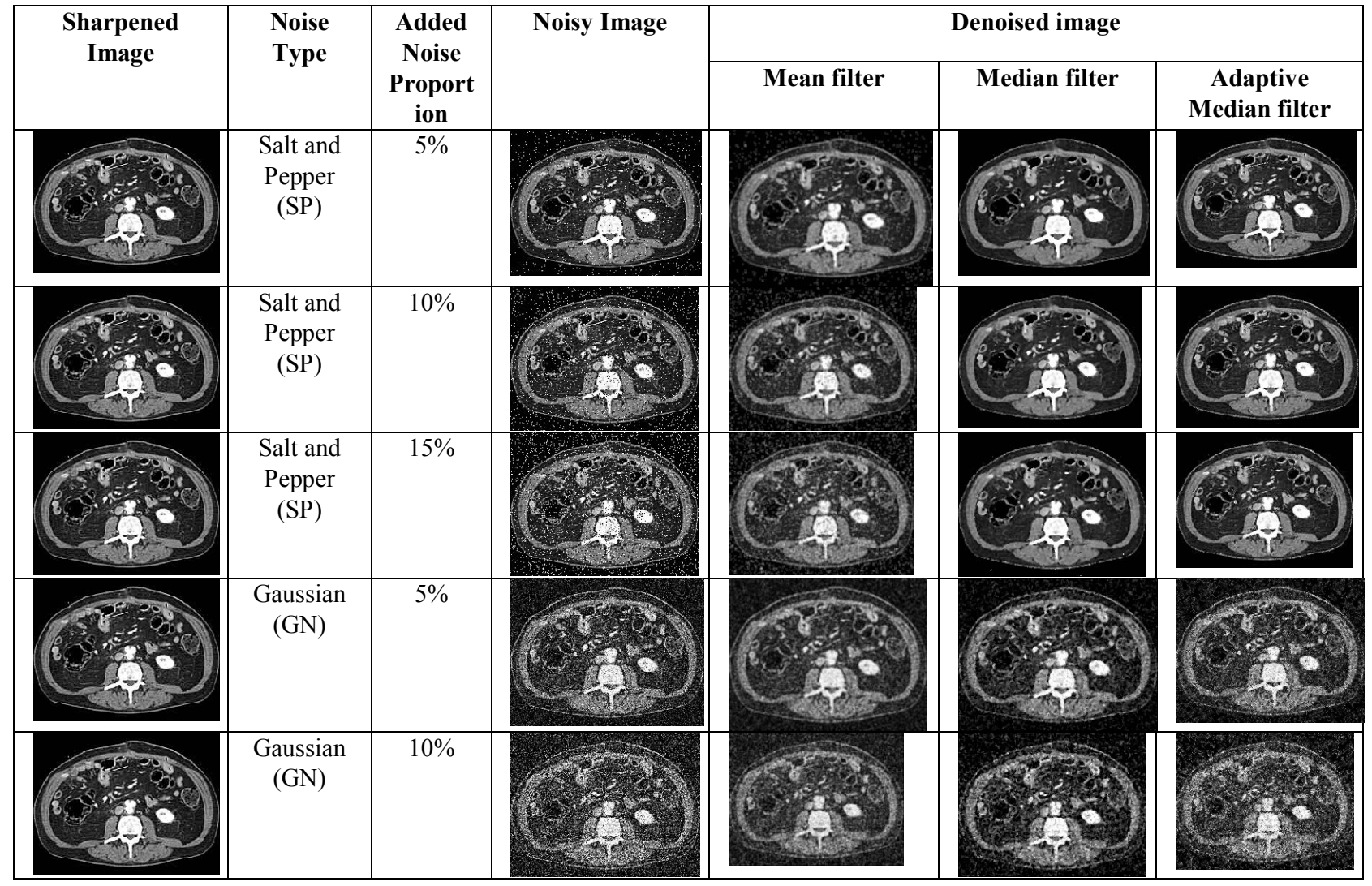


IJCSI International Journal of Computer Science Issues, Volume 14, Issue 1, January 2017

ISSN (Print): 1694-0814 | ISSN (Online): 1694-0784

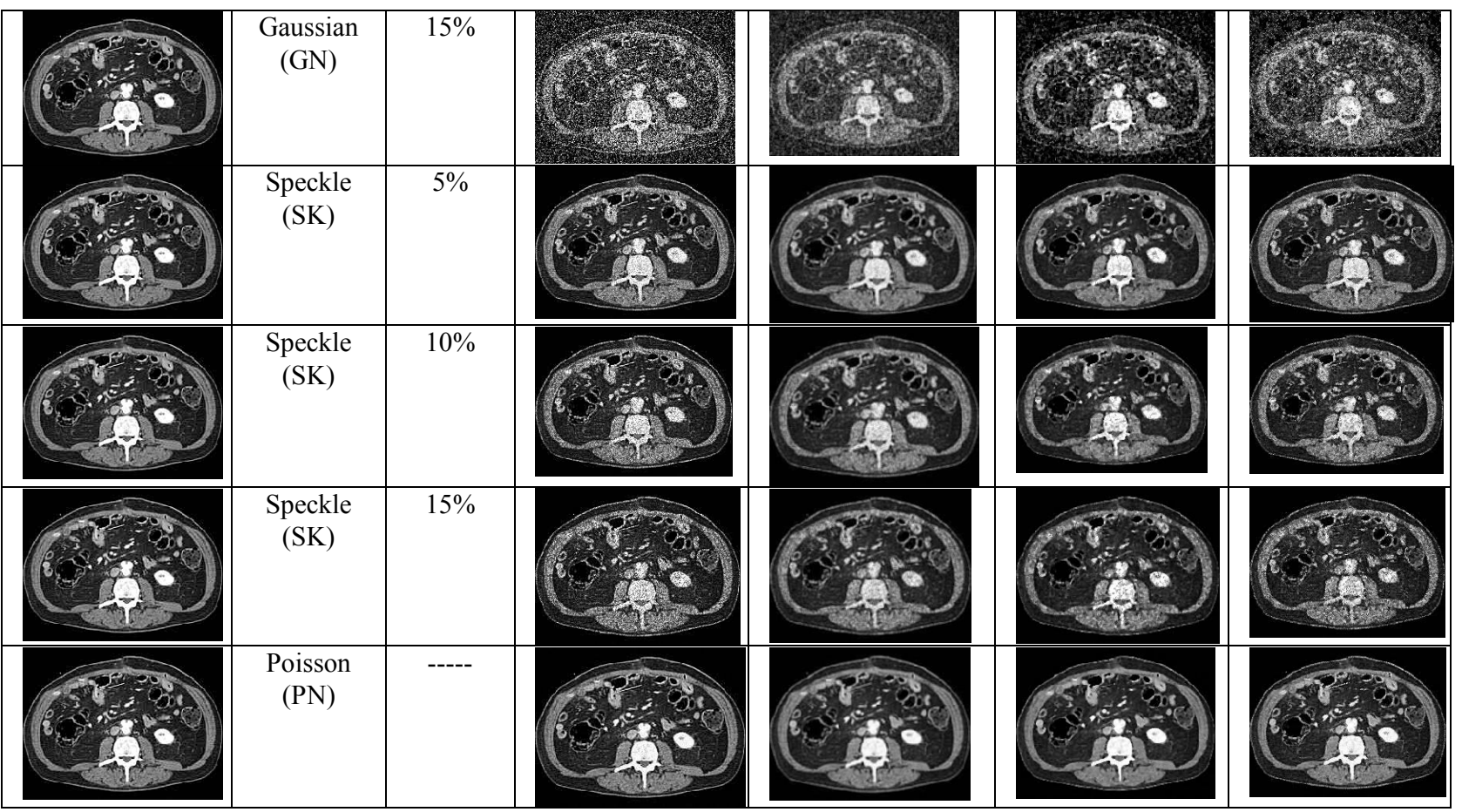

Fig 7 Sharpened image of a sample colon MRI image and corresponding denoised images obtained by using mean, median and adaptive median filters for different noise types and proportions.

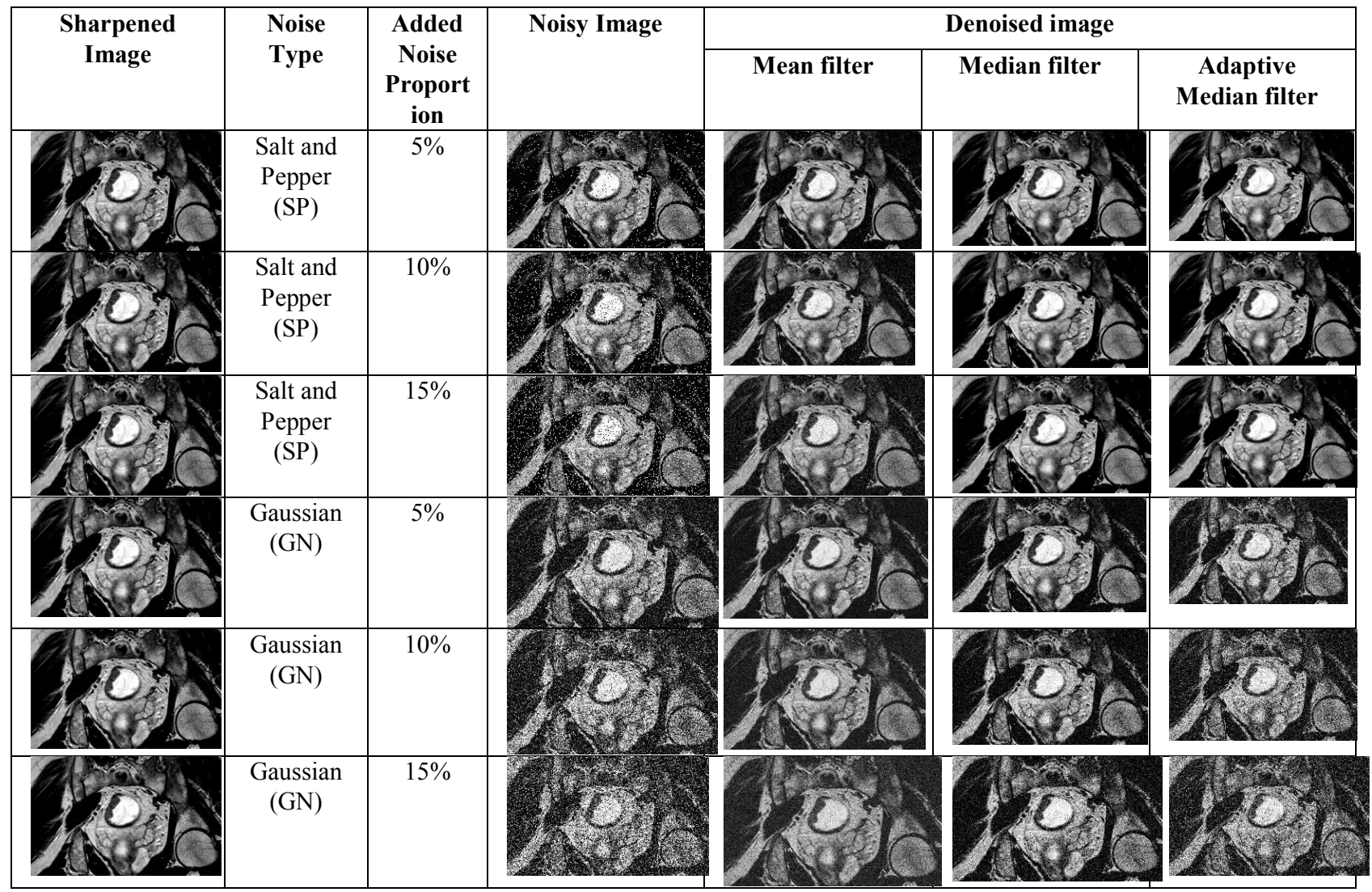




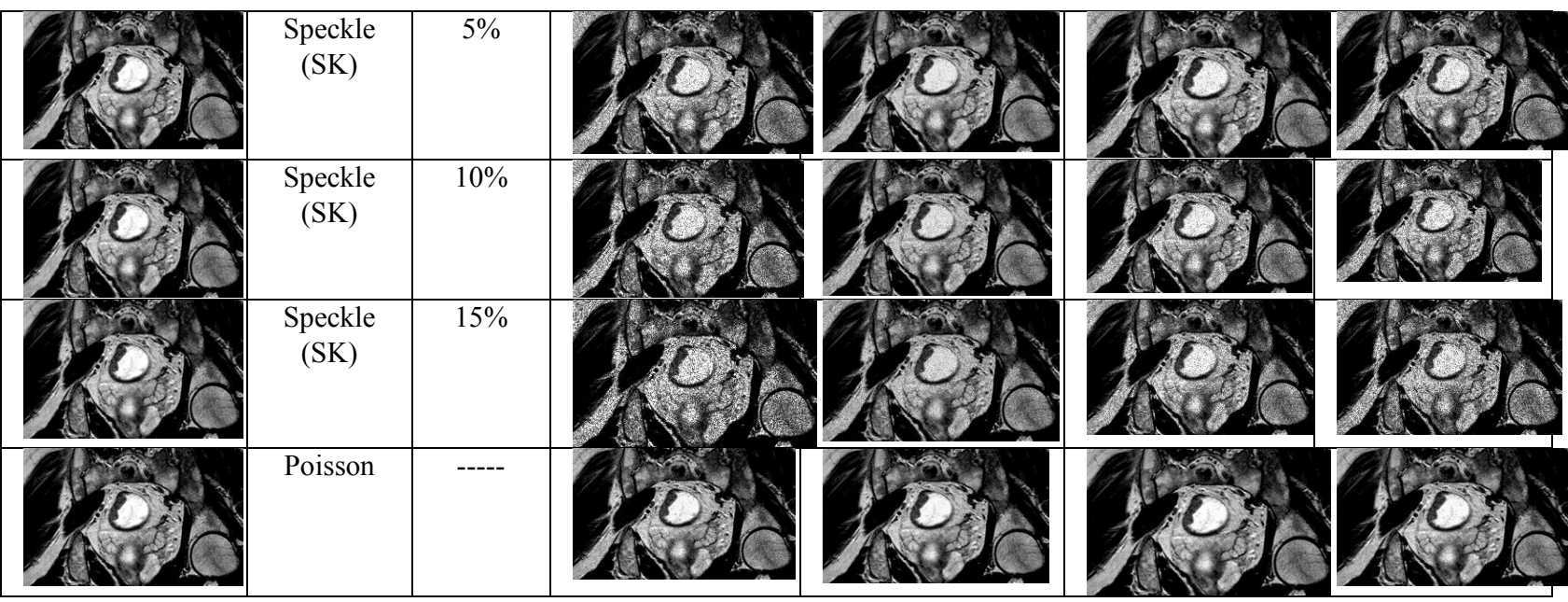

Fig 8 Sharpened image of a sample rectum MRI image and corresponding denoised images obtained by using mean, median and adaptive median filters for different noise types and proportions.

Table 3 : Performance comparison of noise filters in terms of MSE and PSNR values of denoised images colon and rectum for different noise types and proportions-Noise Model(NM), Noise Proportion(NP), Filtering Method(FM).

\begin{tabular}{|c|c|c|c|c|c|c|}
\hline \multirow[t]{2}{*}{ NM } & \multirow{2}{*}{$\begin{array}{l}\text { NP } \\
(\%)\end{array}$} & \multirow[t]{2}{*}{ FM } & \multicolumn{2}{|c|}{ Colon Images } & \multicolumn{2}{|c|}{ Rectal Images } \\
\hline & & & PSNR & MSE & PSNR & MSE \\
\hline SP & 5 & MF & 24.64781 & 0.003662 & 23.53642 & 0.006587 \\
\hline SP & 5 & MDF & 34.1166 & 0.000727 & 32.64571 & 0.006856 \\
\hline SP & 5 & ADMF & 38.18087 & 0.000309 & 36.10664 & 0.006154 \\
\hline SP & 10 & MF & 21.75163 & 0.006865 & 21.07674 & 0.009371 \\
\hline SP & 10 & MDF & 32.66881 & 0.000928 & 31.06208 & 0.006956 \\
\hline SP & 10 & ADMF & 36.7396 & 0.00041 & 34.85967 & 0.006171 \\
\hline SP & 15 & MF & 19.80499 & 0.010624 & 19.42707 & 0.012679 \\
\hline SP & 15 & MDF & 30.49571 & 0.001289 & 28.67322 & 0.007192 \\
\hline SP & 15 & ADMF & 35.24684 & 0.000528 & 33.66746 & 0.006203 \\
\hline GN & 5 & MF & 20.78644 & 0.008466 & 20.45601 & 0.010335 \\
\hline$\overline{\mathrm{GN}}$ & 5 & MDF & 21.4844 & 0.007242 & 19.83983 & 0.012229 \\
\hline GN & 5 & ADMF & 17.5263 & 0.017747 & 16.94425 & 0.020635 \\
\hline GN & 10 & $\mathrm{MF}$ & 18.15007 & 0.015433 & 18.15962 & 0.016204 \\
\hline GN & 10 & MDF & 18.8182 & 0.01325 & 17.67728 & 0.018211 \\
\hline$\overline{\mathrm{GN}}$ & 10 & ADMF & 14.96653 & 0.031923 & 14.62416 & 0.034756 \\
\hline GN & 15 & MF & 16.62497 & 0.021915 & 16.7846 & 0.021739 \\
\hline$\overline{\mathrm{GN}}$ & 15 & MDF & 17.22685 & 0.019068 & 16.34384 & 0.024048 \\
\hline GN & 15 & $\mathrm{ADMF}$ & 13.58839 & 0.043806 & 13.33766 & 0.046598 \\
\hline SK & 5 & MF & 27.3768 & 0.002257 & 25.45608 & 0.005265 \\
\hline SK & 5 & MDF & 26.50544 & 0.002496 & 23.16845 & 0.008411 \\
\hline SK & 5 & ADMF & 23.72444 & 0.004538 & 21.01868 & 0.010007 \\
\hline SK & 10 & MF & 25.36213 & 0.003395 & 23.54946 & 0.006264 \\
\hline SK & 10 & MDF & 24.05114 & 0.004296 & 21.24749 & 0.010135 \\
\hline SK & 10 & ADMF & 21.02295 & 0.008469 & 19.02555 & 0.013802 \\
\hline SK & 15 & $\mathrm{MF}$ & 24.02092 & 0.004528 & 22.36101 & 0.007282 \\
\hline SK & 15 & MDF & 22.53785 & 0.006047 & 20.09648 & 0.011853 \\
\hline SK & 15 & ADMF & 19.42026 & 0.012272 & 17.81784 & 0.017458 \\
\hline $\mathrm{PN}$ & -- & MF & 31.03802 & 0.003393 & 29.72008 & 0.004424 \\
\hline $\mathrm{PN}$ & -- & MDF & 32.37568 & 0.00428 & 28.91812 & 0.006963 \\
\hline PN & -- & ADMF & 31.30535 & 0.008426 & 26.86018 & 0.006589 \\
\hline
\end{tabular}


Table 4 : The optimal noise filters for different noise models

\begin{tabular}{|l|l|l|}
\hline \multirow{2}{*}{$\begin{array}{c}\text { Noise } \\
\text { model }\end{array}$} & \multicolumn{2}{c|}{ Filtering method } \\
\cline { 2 - 3 } & Colon image & Rectum image \\
\hline SP & ADMF & ADMF \\
\hline GN & MDF & MF \\
\hline SK & MF & MF \\
\hline PN & MDF & MF \\
\hline
\end{tabular}

\section{Conclusion}

In this paper, the preprocessing of MRI images of colon and rectum is investigated by considering various methods of digital image enhancement, image sharpening and image denoising. The suitable methods that yield optimal results in terms of different image quality metrics are determined. The experimentation has been carried out on the MRI images of colon and rectum showing incidence of various stages of cancer. It is observed that contrast adjustment technique for image enhancement, which is further sharpened using high pass filtering, yields good quality image. Further, the noise filters, namely, mean, median and adaptive median filters, are employed to denoise the sharpened image and obtain better quality image. The proposed preprocessing yields a clear image without any artifacts and at the same time without loss of any information. The outcome of the present investigation is helpful in assisting precision diagnosis of abnormality or finding the stage of colorectal cancer.

\section{References}

[1] Shameena N., Rahna Jabbar, "A Study of Preprocessing and Segmentation Techniques on Cardiac Medical Images", International Journal of Engineering Research and Technology (IJERT), Vol. 3, No. 4, 2014, pp. 336-341.

[2] Anithadevi D., Perumal K., "Novel Approach for Noise Removal of Brain Tumor MRI Images", Journal of Biomedical Engineering and Medical Imaging (JBEMi), Vol. 2, No. 3, 2015, pp. 1-14.

[3] Priyanka Kamboj., Versha Rani., "A Brief Study of Various Noise Model and Filtering Techniques", Journal of Global Research in Computer Science(JGRCS), Vol. 4, No. 4, 2013, pp. 166-171.

[4] Syed Amjad Ali., Srinivasan Vatsal., K. Lalkishore., "An Efficient Denoising Technique for CT Images using Windowbased Multi-Wavelet Transformation and Thresholding", European Journal of Scientific Research, Vol. 48, No. 2, 2010, pp. 315-325.
[5] Neha Jain., D. S. Karaulia., “A Comparative Analysis of Filters on Brain MRI Images", International Journal of Advanced Research in Computer Science and Software Engineering (IJARCSSE), Vol. 4, No. 11, 2014, pp. 893-897.

[6] S. Valarmathy., R. Ramani., R. N. Suthanthira Vanitha., "A Survey of Recent Image Segmentation Techniques for MRI Brain Images", International Journal of Computer Science and Technology (IJCST), Vol. 4, No. 1, 2013, pp. 241-245.

[7] P.Geetha., V.Selvi., "An Impression of Cancers and Survey of Techniques in Image Processing for Detecting Various Cancers: A Review", International Research Journal of Engineering and Technology (IRJET), Vol. 2, No. 9, 2015, pp. 236-242.

[8] Priyadharsini.B., "A Novel Noise Filtering Technique for Denoising MRI Images", International Journal of Innovative Research in Computer and Communication Engineering (IJIRCCE), Vol. 2, Special Issue1, 2014, pp. 2428-2433.

[9] S. Jacily Jemila., T. Jayasankar., "An Automated Cancer Recognition System for MRI Images Using Neuro Fuzzy Logic", International Journal of Computer Information Systems, Vol. 2, No. 5, 2011, pp. 18-22.

[10] Rajesh C. Patil., A. S. Bhalchandra., "Brain Tumour Extraction from MRI Images Using MATLAB", International Journal of Electronics, Communication Soft Computing Science and Engineering (IJECSCSE), Vol. 2, No. 1, 2012, pp. 1-4.

[11] Siti Noraini Sulaiman., Siti Mastura CheIshak., Iza Sazanita Isa., Norhazimi Hamzah., "Denoising of Noisy MRI Brain Image by Using Switching-based Clustering Algorithm", International Conference on Control System, Computing and Engineering(ICCSCE), 2014, pp. 1-6.

[12] N.Gopinath., "Extraction of Cancer Cells from MRI Prostate Image Using MATLAB", International Journal of Engineering Science and Innovative Technology (IJESIT), Vol. 1, No. 1, 2012, pp. 27-35.

[13] Anuradha S. Deshpande., Dhanesh D. Lokhande., Rahul P. Mundhe., Juilee M.Ghatole., "Lung Cancer Detection with fusion of CT and MRI Images using Image Processing", International Journal of Advanced Research in Computer Engineering and Technology (IJARCET), Vol. 4, No. 3, 2015, pp. 763-767.

[14] E. Ben George., M.Karnan., "MRI Brain Image Enhancement using Filtering Techniques", International Journal of Computer Science \& Engineering Technology (IJCSET), Vol. 3, No. 9, 2012, pp. 399-403.

[15] Deepa., M. G. Sumithra., "MRI Medical Image Denoising by Combined Spectral Subtraction and Wavelet Based Methods", ARPN Journal of Engineering 
and Applied Sciences, Vol. 10, No. 4, 2015, pp. 18151821.

[16] P. Deepa., M.Suganthi., "Performance Evaluation of Various Denoising Filters for Medical Image", International Journal of Computer Science and Information Technologies (IJCSIT), Vol.3, No. 5, 2014, pp. 4205-4209.

[17] Bhausaheb Shinde., Dnyandeo Mhaske., A.R. Dani., "Study of Noise Detection and Noise Removal Techniques in Medical Images", International Journal of Image, Graphics and Signal Processing, Vol. 2, 2012, pp. 51-60.

[18] Amutha .S., Ramesh Babu D.R., Ravi Shankar., Harish Kumar.N., "MRI Denoising and Enhancement Based on Optimized Single-Stage Principle Component Analysis", International Journal of Advances in Engineering \& Technology (IJAET), Vol.5, No. 2, 2013, pp. 224-230.

[19] Pratik Vinayak Oak., R. S. Kamathe., "Contrast Enhancement of Brain MRI images using Histogram Based Techniques", International Journal of Innovative Research in Electrical, Electronics, Instrumentation and Control Engineering( IJIREEICE ), Vol. 1, No. 3, 2013, pp. $90-94$.

[20] Kishor Kumar Reddy C., Anisha P. R., Narasimha Prasad L. V., "A Novel Approach for Detecting the Bone Cancer and its Stage based on Mean Intensity and Tumor Size", Recent Researches in Applied Computer Science, 2015, pp. 162-171.

[21] Arjun Nelikanti., Narasimha Prasad L V., Naresh Goud M., "Colorectal Cancer MRI Image Segmentation Using Image Processing Techniques", International Journal on Computer Science and Engineering (IJCSE), Vol. 6, No. 7, 2014, pp. 280-286.

[22] Harish Kumar.N., Amutha. S., Ramesh Babu .D. R., "Enhancement of Mammographic Images using Morphology and Wavelet Transform", International Journal of Computer Technology and Applications (IJCTA), Vol. 3, No. 1, 2012, pp. 192-198.

[23] V.Velusamy., M.Karnan., R.Sivakumar., N.Nandhagopal., "Enhancement Techniques and Methods for MRI- A Review", International Journal of Computer Science and Information Technologies (IJCSIT), Vol. 5 , No. 1 , 2014, pp. 397-403.

[24] Huanshuang Niu., Zhuoyong Zhang., Yuhong Xiang., Liting Zhao., Fan Yang., "Evaluation of Signal Preprocessing Methods for the Detection of Endometrial Cancer by using Near Infrared Spectroscopy", Journal of Theoretical and Applied Information Technology (JATIT), Vol. 50, No. 1, 2013, pp. 12-21.

[25] D. Belsare., M. M. Mushrif., "Histopathological Image Analysis using Image Processing Techniques: An
Overview", Signal \& Image Processing : An International Journal (SIPIJ), Vol. 3, No. 4, 2012, pp. 2336 .

[26] KimmiVerma., Aru Mehrotra., Vijayeta Pandey., Shardendu Singh., "Image Processing Techniques for the Enhancement of Brain Tumor Patterns", International Journal of Advanced Research in Electrical, Electronics and Instrumentation Engineering (IJAREEIE), Vol. 2, No. 4, 2013, pp. 1611-1615.

[27] Atiyeh Hashemi., Abdol Hamid Pilevar., Reza Rafeh., Mass Detection in Lung CT Images using Region Growing Segmentation and Decision Making Based on Fuzzy Inference System and Artificial Neural Network, International Journal of Image, Graphics and Signal Processing (IJIGSP), Vol. 6, 2013, pp. 16-24.

[28] Sheela V.K., S. Suresh Babu., "Preprocessing Techniques for Brain Tumor Detection and Segmentation", International Research Journal of Engineering and Technology (IRJET), Vol. 2, No. 3, 2015, pp. 1208-1212. 